\section{A) Check for updates}

Cite this: Org. Chem. Front., 2021, 8 2932

Received 15th February 2021,

Accepted 29th March 2021

DOI: $10.1039 / \mathrm{d} 1 \mathrm{qo} 00259 \mathrm{~g}$

rsc.li/frontiers-organic

\title{
Organo-redox-catalysis for the difunctionalization of alkenes and oxidative Ritter reactions by $\mathrm{C}-\mathrm{H}$ functionalization $\uparrow$
}

\begin{abstract}
Sensheng Liu and Martin Klussmann (iD *
Transition metals are the dominant catalysts for redox-reactions between peroxides and organic substrates. Here, we show that triarylamines can act as organic redox-catalysts, enabling oxidative difunctionalization reactions of alkenes and oxidative Ritter-reactions. Styrene derivatives can be functionalized with alkyl radicals, generated from plain and halogenated hydrocarbons, and with nucleophiles, including nitriles, acetic acid, alcohols and fluoride. An oxidative Ritter reaction can be conducted between allylic $\mathrm{C}-\mathrm{H}$ bonds as well as fluorene and acetonitrile. Benzoyl peroxide is the oxidant in both reactions. Mechanistic studies suggest that the triarylamines are catalysts and not initiators, mediating the reaction by electron transfer to the peroxide, forming benzoyloxyl radicals, and from C-radical intermediates, forming carbocations.
\end{abstract}

\section{Introduction}

The difunctionalization of alkenes is a powerful method for the construction of $\mathrm{C}-\mathrm{C}$ and $\mathrm{C}-\mathrm{X}$ bonds. ${ }^{1-4} \mathrm{~A}$ very interesting type amongst those is the successional addition of a radical and a nucleophile. ${ }^{1,3}$ This method enables functionalizing olefins with a wide variety of reagents in a selective manner, given that radicals and nucleophiles generally react complementarily. However, these reactions are still lacking a truly broad substrate scope, spurring our efforts of further research.

A widely used strategy for generating radicals from simple substrates is $\mathrm{C}-\mathrm{H}$ bond cleavage by hydrogen atom transfer (HAT) to oxyl radicals, which are readily generated from peroxides. ${ }^{5}$ The subsequent addition of a nucleophile requires an electron transfer (ET) step to convert the radical intermediate into a carbocation. Thus, transition metals are widely used as redox-catalysts in such reactions, as they can mediate peroxide $\mathrm{O}-\mathrm{O}$ bond cleavage and subsequent ET (Scheme 1a). ${ }^{1,3,6-9}$

Alternative methods for the consecutive addition of radicals and nucleophiles utilize organic photocatalysts, ${ }^{10,11}$ hypervalent iodine reagents or iodide as catalyst ${ }^{12-14}$ and electrochemistry. ${ }^{15,16}$ We are not aware, however, of the use of an organo-redox catalyst independent of irradiation in such reactions. Here, we report the use of triarylamines as catalysts

Max-Planck-Institut für Kohlenforschung, Kaiser-Wilhelm-Platz 1, 45470 Mülheim an der Ruhr, Germany.E-mail: klusi@mpi-muelheim.mpg.de

$\dagger$ Electronic supplementary information (ESI) available: Method development, synthesis, characterization and mechanistic studies. See DOI: 10.1039/ d1qo00259g in the activation of peroxides for synthetic radical reactions (Scheme 1b).

Triarylamines can form stable ammoniumyl radical cation salts by ET, and variation of the aryl-substituents allows for fine-tuning of their properties. ${ }^{17-21}$ Both the amines and the radical cations are widely applied in electro-optical materials. ${ }^{18-20,22}$ The radical cations can be used as stoichiometric single-electron oxidants in chemical reactions, ${ }^{23}$ or in substoichiometric amounts as initiators of radical chain

a) Radical reactions by transition metal-catalyzed redox-activation of peroxides:

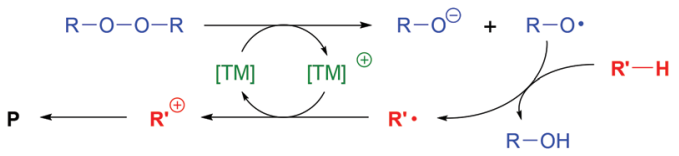

b) This work: organo-redox-catalysis
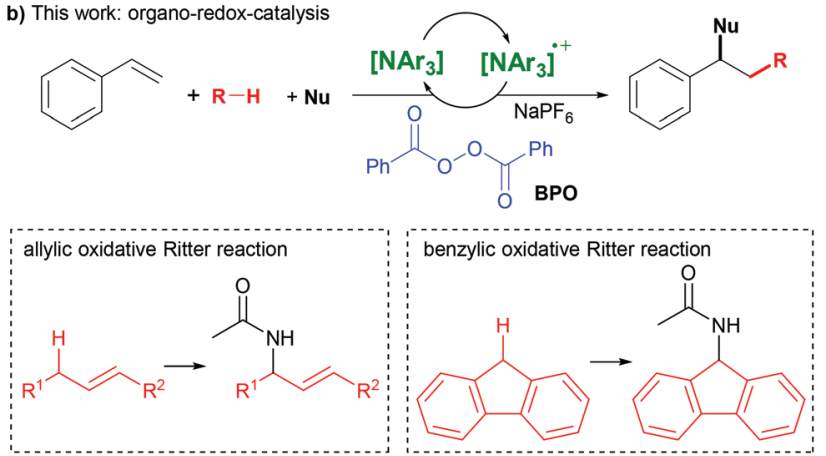

Scheme 1 Activation of peroxides and formation of carbocations by redox-catalysis. 
reactions. ${ }^{17,24}$ Both the amines and the radical cations are also utilized as redox-catalysts in electrochemical ${ }^{25,26}$ or photochemical reactions, ${ }^{27,28}$ as well as in aerobic oxidations. ${ }^{29,30}$ Despite this plethora of applications, we are not aware of amine-based redox catalysis in the activation of peroxides, which would open many opportunities for synthetic applications.

$N, N$-Dialkylanilines like $\mathbf{1}$ are well-known to generate radicals from diacylperoxides, especially benzoyl peroxide (BPO, Scheme $2 \mathrm{a}) .{ }^{31,32}$ The reaction is irreversible due to the reactivity of the ammoniumyl radical cation 2 , which readily forms a C-radical 3, an iminium ion $\mathbf{4}$ and other products derived thereof. $^{32,33}$ In contrast, we assumed that triarylamines $\mathbf{A}$ could be suitable candidates for catalysis, as the stable radical cation salts A+ could be regenerated by ET (Scheme $2 \mathrm{~b}$ ).

We had previously utilized BPO in the addition of thioxanthene and similarly facile radical precursors together with nucleophiles to styrenes, which was rationalized as a radicalchain reaction. ${ }^{34}$ The addition of hexafluorophosphoric acid $\left(\mathrm{HPF}_{6}\right)$ was found to modulate the redox potential of BPO, and the addition of $N, N$-dimethylanilines as initiators allowed difunctionalization with acetonitrile with moderate success. We kept working on finding a more efficient method for a broad substrate scope that would also avoid the use of a strong acid.

\section{Results and discussion}

We found that the addition of cyclohexane and acetonitrile to styrene (5a) took place in the presence of catalytic amounts of some triarylamines and $\mathrm{NaPF}_{6}$ as an additive, forming the desired product $\mathbf{6 a}$ in good yields (Table 1 , entries 1-3). The most effective amine was 4-iodo- $N, N$-diphenylaniline (A1), closely followed by tris(4-methylphenyl)amine (A2) and tris(4bromophenyl)amine (A3), other amines were much less efficient. Without triarylamine, product $\mathbf{6 a}$ was not formed (entry 4) and the addition of $\mathrm{NaPF}_{6}$ is indispensable (entry 5). A reduction in the product's yield was also seen with other

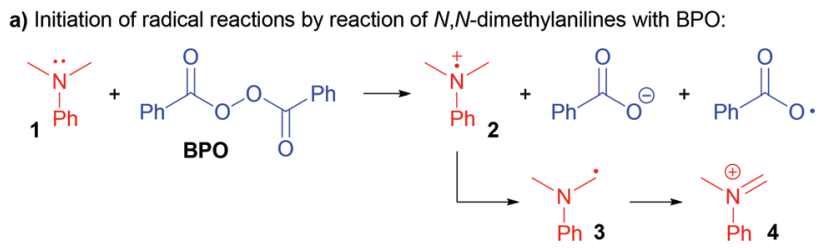

b) Potential re-activation of triarylamines by electron transfer (ET):

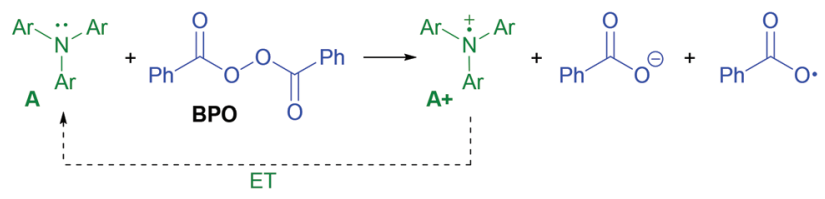

Scheme 2 Activation of BPO with N,N-dimethylaniline and triarylamines.
Table 1 Evaluation of catalysts ${ }^{a}$

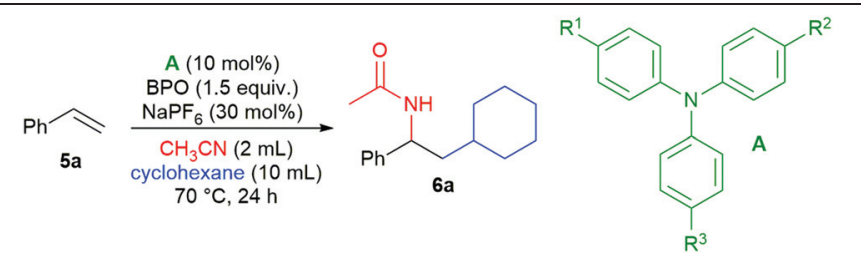

\begin{tabular}{lllllll}
\hline Entry & Catalyst & $\mathrm{R}^{1}$ & $\mathrm{R}^{2}$ & $\mathrm{R}^{3}$ & Additive & Yield $^{b}(\%)$ \\
\hline 1 & $\mathbf{A 1}$ & $\mathrm{I}$ & $\mathrm{H}$ & $\mathrm{H}$ & $\mathrm{NaPF}_{6}$ & $91(86)$ \\
2 & $\mathbf{A} 2$ & $\mathrm{Me}$ & $\mathrm{Me}$ & $\mathrm{Me}$ & $\mathrm{NaPF}_{6}$ & 58 \\
3 & $\mathbf{A} 3$ & $\mathrm{Br}$ & $\mathrm{Br}$ & $\mathrm{Br}$ & $\mathrm{NaPF}_{6}$ & 86 \\
4 & - & & & & $\mathrm{NaPF}_{6}$ & 0 \\
5 & $\mathbf{A 1}$ & $\mathrm{I}$ & $\mathrm{H}$ & $\mathrm{H}$ & - & 0
\end{tabular}

${ }^{a}$ Reaction conditions: $5 a(0.5 \mathrm{mmol}), \mathbf{A}(0.05 \mathrm{mmol}, 10 \mathrm{~mol} \%), \mathrm{CH}_{3} \mathrm{CN}$ ( $2 \mathrm{~mL})$, cyclohexane $(10 \mathrm{~mL})$, BPO $(0.75 \mathrm{mmol}, 1.5$ equiv. $)$, additive (0.15 mmol, 0.3 equiv.). ${ }^{b}$ Determined by ${ }^{1} \mathrm{H}$ NMR spectroscopic analysis of the crude reaction mixture relative to internal standard $1,3,5$ trimethoxybenzene, isolated yield in parentheses.

additives and oxidants (see the ESI $\uparrow$ for a detailed investigation). The product's structure supported the subsequent addition of a cyclohexyl radical and acetonitrile as a nucleophile in a Ritter reaction.

With these reaction conditions, we investigated the product scope by testing other substrates. Using cyclopentane, cyclohexane, cycloheptane, and cyclooctane as radical precursors with acetonitrile as nucleophile afforded the products $\mathbf{6 a - 6} \mathbf{d}$ in good yields of $80-86 \%$ (Scheme 3). Methylcyclohexane gave a mixture of regioisomers from which we could isolate $6 \mathbf{e}$, the major one, in $34 \%$ yield. With $n$-hexane, a mixture of the iso-

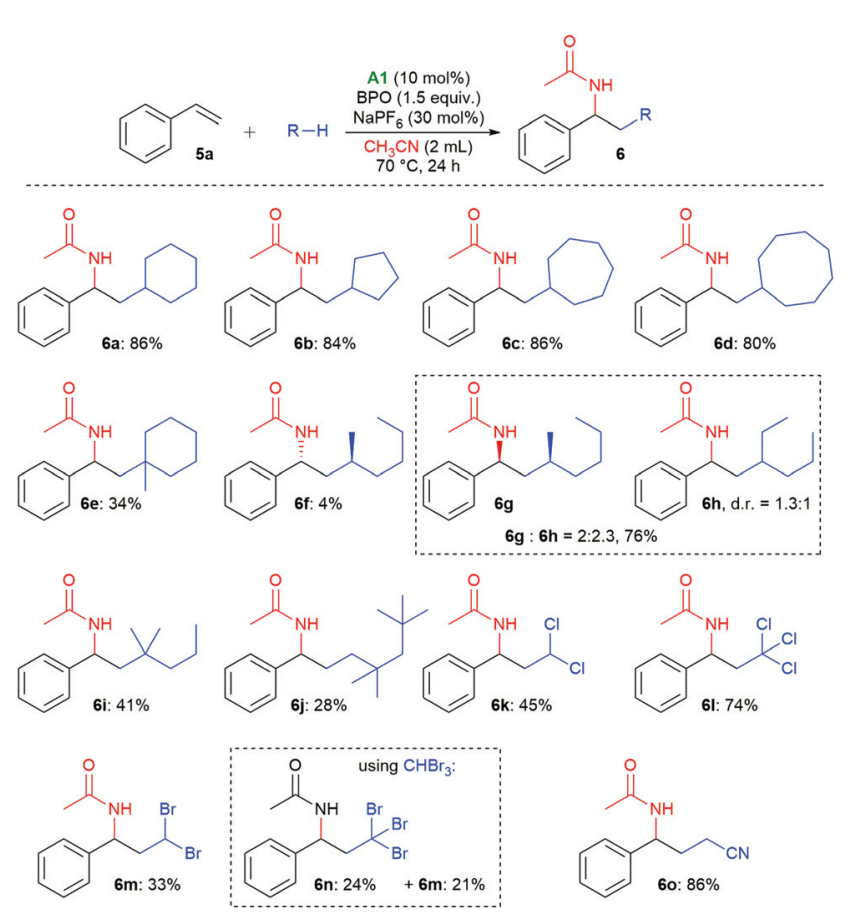

Scheme 3 Scope of radicals. 
meric products $\mathbf{6 f}-\mathbf{6 h}$ was isolated in $80 \%$ overall yield, from which we could isolate the isomer 6 f in $4 \%$ yield as a pure compound by column chromatography. With 2-methylpentane, the selectivity for the tertiary $\mathrm{C}-\mathrm{H}$ bond was relatively high, allowing for isolation of the major isomer $6 \mathbf{i}$ in $41 \%$ yield. With 2,2,4,4-tetramethylpentane, only isomer $\mathbf{6 j}$ was isolated, apparently because the methylene group is sterically shielded, resulting in HAT from a primary $\mathrm{C}-\mathrm{H}$ bond. The haloalkanes dichloromethane, chloroform, dibromomethane and bromoform could also be employed successfully in this reaction, producing the products $6 \mathbf{k}-\mathbf{6 m}$. When using bromoform, not only $\mathbf{6 n}$ was formed by HAT, but also $\mathbf{6 m}$ by bromine atom transfer. ${ }^{35,36}$ When only acetonitrile was used as solvent, 60 was isolated in $86 \%$.

Styrenes with various substituents on the aromatic ring afforded the desired products in generally good yields (Scheme 4). There is no clear electronic substituent effect on the product yields, also substitution in the ortho position was not detrimental (7l-7n). Only in the case of $p$-methoxystyrene, the desired product was only observed in traces (7b). 2-Methylstyrene and stilbene could also be employed, giving
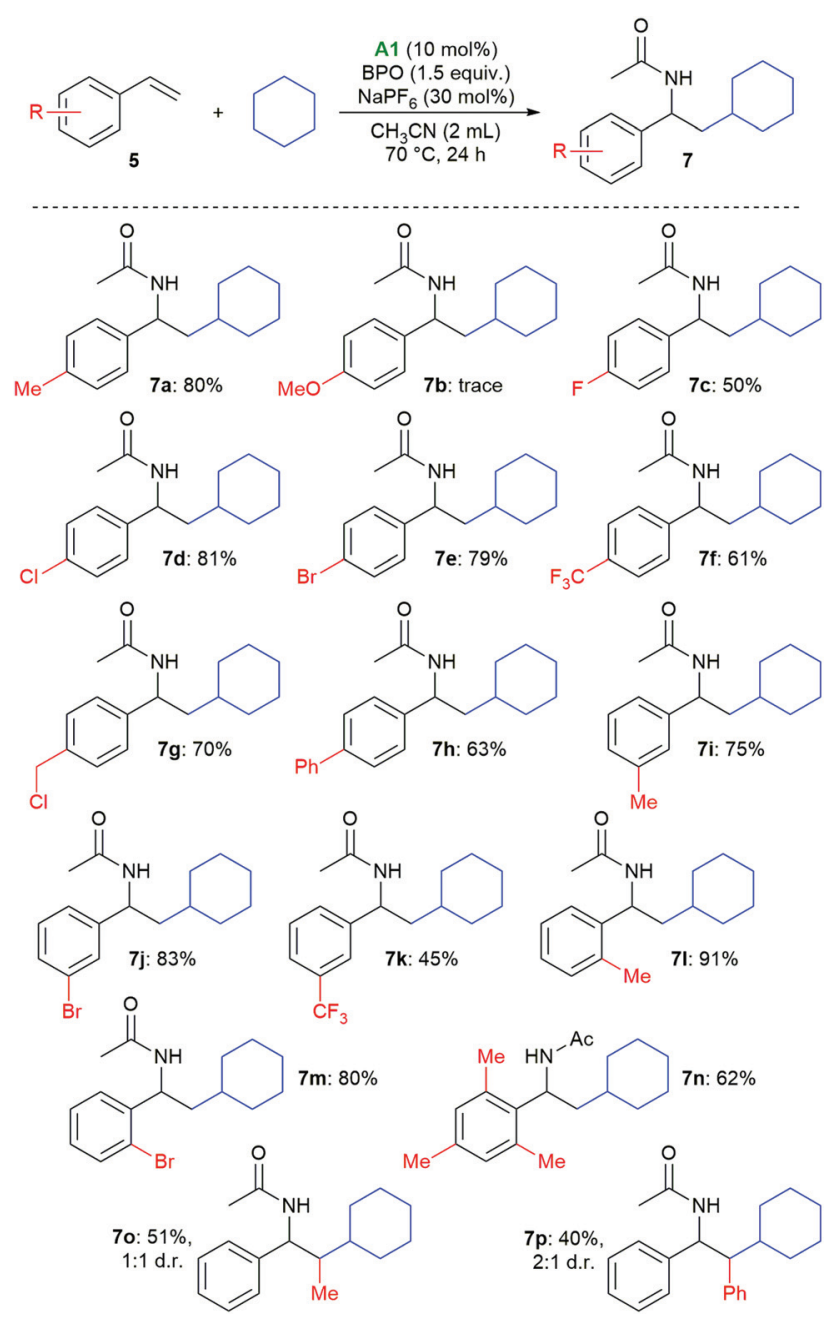

Scheme 4 Substrate scope of substituted styrenes. the expected products $7 \mathbf{o}$ and $7 \mathbf{p}$ in medium yields and as mixtures of diastereomers.

Next, the scope with respect to nucleophiles was explored. As shown in Scheme 5a, different nitrile solvents and cyclohexane delivered the desired amides $\mathbf{8 a}-\mathbf{8 e}$ in good yields of $48 \%-76 \%$. Similarly, when acetic acid was used, the corresponding acetate $8 \mathbf{f}$ was formed in 63\% yield. With 1,1diphenylethylene, difunctionalization with the $\alpha$-cyanoalkyl radical from acetonitrile and alcohols was possible. With methanol and ethanol, the products $\mathbf{8 g}$ and $\mathbf{8 h}$ were isolated in $51 \%$ and $41 \%$ yields, respectively, however, long-chain alcohols showed a low reactivity. Very similar tertiary alcohols had recently been synthesized by copper-catalysis at higher temperature. ${ }^{37}$ We also isolated the dimer 9 from unsuccessful tests of other nucleophiles, supporting the occurrence of radical intermediate $\mathbf{1 5} .^{38,39}$ In nitromethane as solvent, phenyl groups were incorporated into the products (10a-10c, Scheme 5b). These likely originated from phenyl radicals, formed by decarboxylation of the benzoyloxyl radicals. Acetonitrile and methanol could be used as nucleophiles, and with triethylamine hydrofluoride even fluoride, albeit in low yield (10c).

The organo-redox system also proved to catalyze the oxidative Ritter reaction of allylic and benzylic $\mathrm{C}-\mathrm{H}$ bonds; for
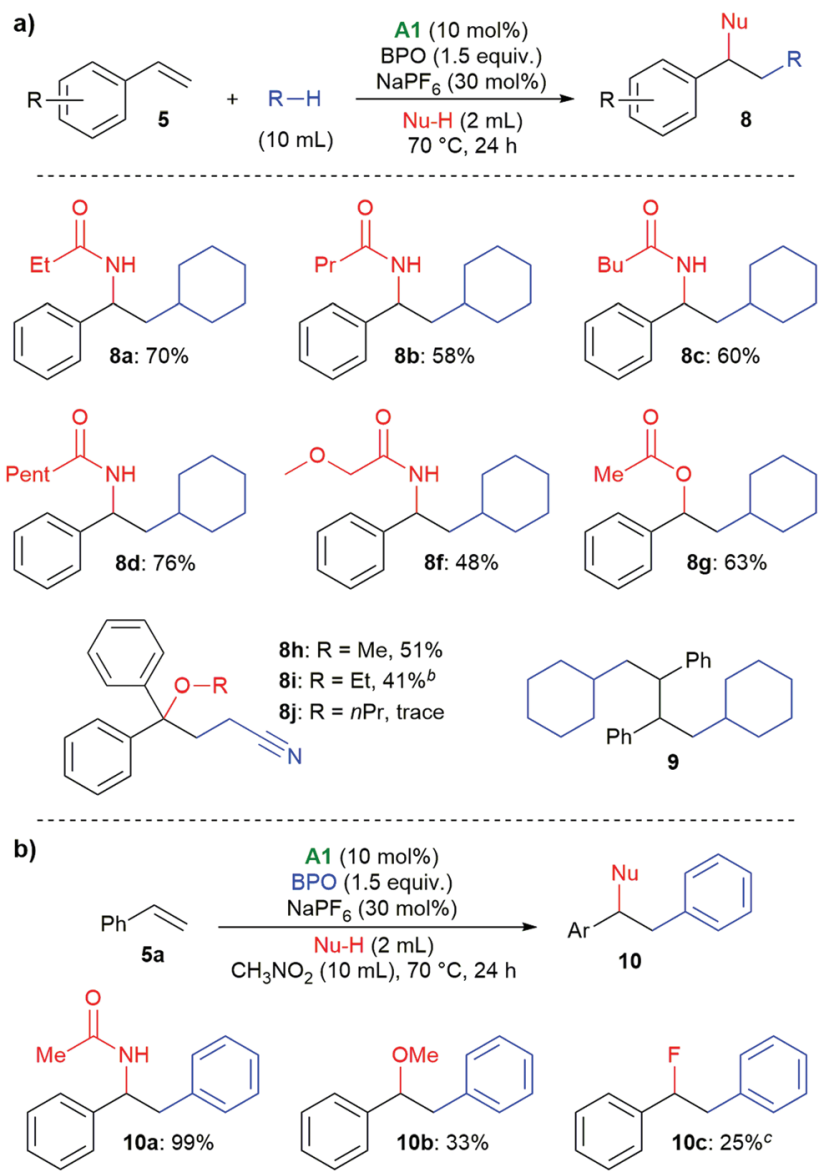

Scheme 5 Scope of nucleophiles. 
a)
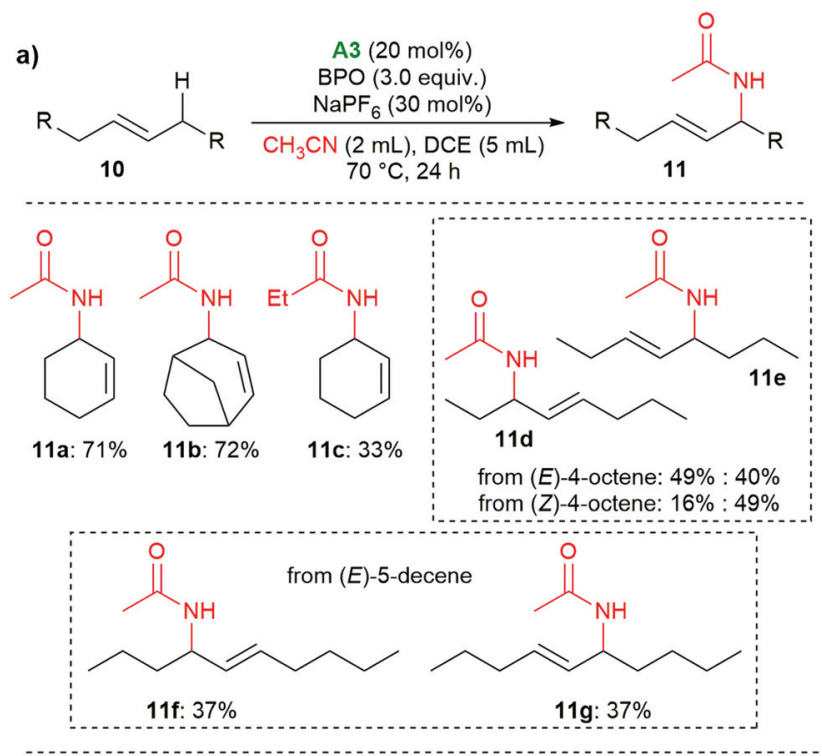

b)
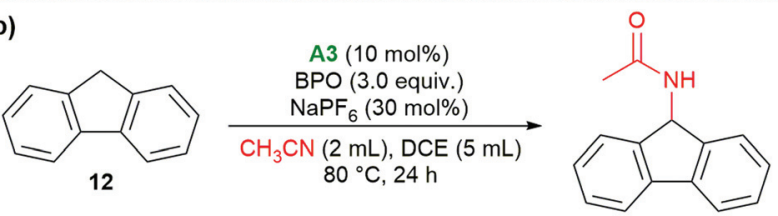

13a: $70 \%$

Scheme 6 Amination of allylic and benzylic $\mathrm{C}-\mathrm{H}$ bond.

these reactions, amine $\mathbf{A} \mathbf{3}$ was found to be superior to $\mathbf{A 1}$ (see the ESI $†$ for details). As shown in Scheme 6a, cyclohexene and bicyclo[3.2.1] oct-2-ene delivered the amides 11a and 11b in $71 \%$ and $72 \%$ yield, respectively. When propionitrile was used, the corresponding product 11c was isolated in 33\% yield. With $(E)$-4-octene, 11d and 11e were isolated in $89 \%$ yield as a mixture of two isomers with a ratio of $1: 0.8$. When $(Z)-4$ octene was used, the same products were isolated with a ratio of $1: 3$. (E)-5-Decene gave the $E$-isomers $\mathbf{1 1 f}$ and $\mathbf{1 1 g}$ in equimolar amounts. When we used $9 H$-fluorene, the benzylic methylene group was functionalized, providing the amide $\mathbf{1 3}$ in $70 \%$ yield (Scheme $6 \mathrm{~b}$ ).

Are the triarylamines efficient initiators of a radical chain reaction, reacting irreversibly, or are they catalysts, achieving turnover ${ }^{40}$ As mentioned above, $N, N$-dialkylanilines are wellknown activators of benzoyl peroxide in radical chain reactions; ${ }^{31}$ however, they proved to be inefficient for the reactions presented here (Scheme 7a). Also, the reaction did not proceed when employing the well know radical initiator azobisisobutyronitrile (AIBN), suggesting that a radical chain mechanism is not operating. We used two radical cation salts, $\left[(\mathbf{A} 1+) \mathrm{SbF}_{6}{ }^{-}\right]$ and $\left[(\mathbf{A} 3+) \mathrm{SbCl}_{6}^{-}\right]$, in place of the corresponding triarylamines under otherwise unchanged reaction conditions (Scheme $7 \mathrm{~b}$ ). In both cases, comparable yields of 6 a were achieved ( $89 \%$ and $84 \%$, resp.). When A3+ was used, its dark blue colour disappeared and $10 \%$ of the reduced form $\mathbf{A} 3$ could be isolated, ruling out that the triarylamines are irreversibly consumed and supporting that both the amine and the oxidized radical cation salts are involved in a catalytic reaction.

a)<smiles>C=Cc1ccccc1</smiles>

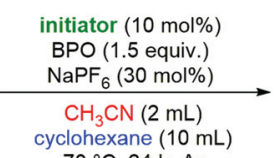<smiles>CC(=O)NC(CC1CCCCC1)c1ccccc1</smiles>

$70^{\circ} \mathrm{C}, 24 \mathrm{~h}, \mathrm{Ar}$
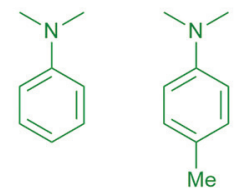

6a: $0 \%$

6a: trace
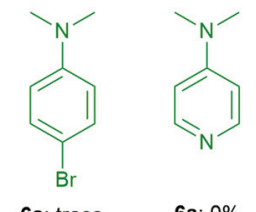

6a: $0 \%$

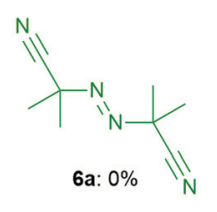

b)
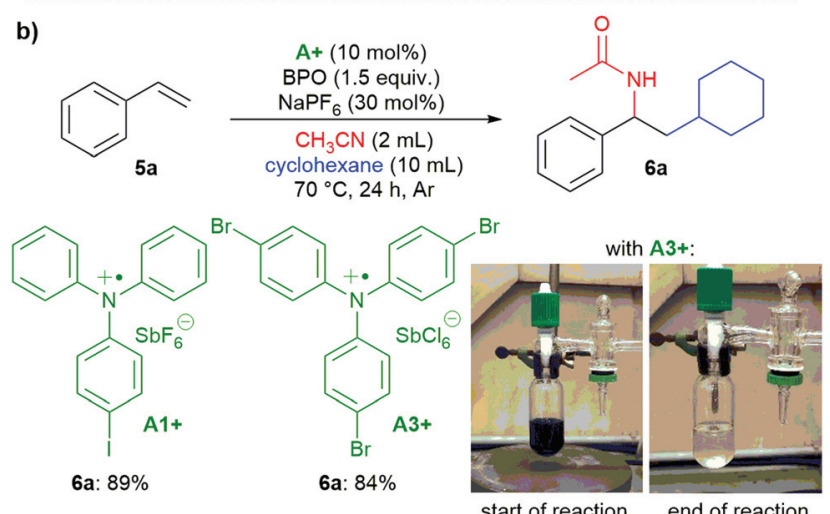

Scheme 7 a) Testing $N, N$-dimethylanilines and AIBN as initiators; (b) triarylamine radical cation salts act as catalysts.

After completion of the reaction, the system remains active. When we added another batch of BPO and styrene to a reaction mixture forming $\mathbf{6 a}$ that had gone to completion, a further $85 \%$ of these added substrates were converted to $\mathbf{6 a}$ after another 30 hours. Also, a third batch of substrates could be converted to product, which further supports that the reaction is catalytic in nature (see the ESI $\dagger$ for details).

We also investigated the system by ${ }^{1} \mathrm{H}-\mathrm{NMR}$ at the reaction temperature of $70{ }^{\circ} \mathrm{C}$ (Fig. 1). Ca. $40 \%$ of BPO on its own had

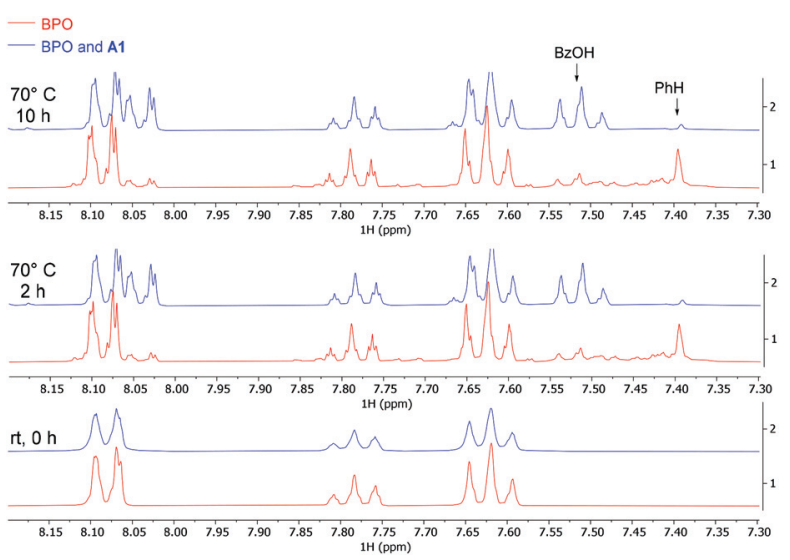

Fig. 1 NMR experiments, comparing thermal and A1-induced BPO decomposition (red and blue lines, resp.); conditions: BPO $(0.1 \mathrm{mmol})$, A1 (10 mol\%), $\mathrm{CD}_{3} \mathrm{CN}(0.5 \mathrm{~mL}), 70^{\circ} \mathrm{C}$. 
a)

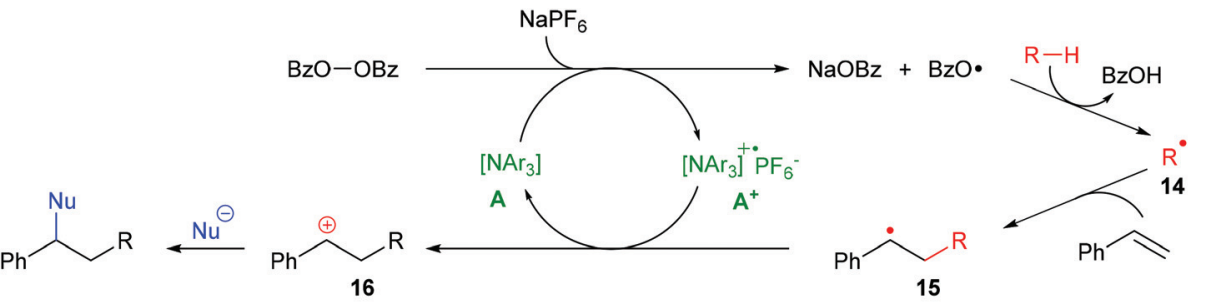

b)
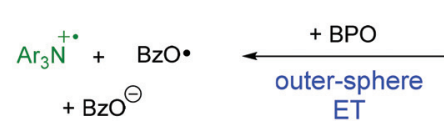

$\mathrm{NAr}_{3}$
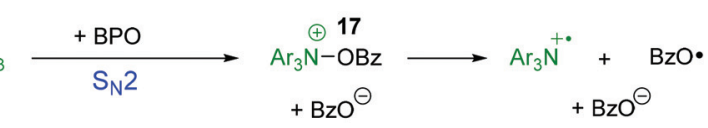

Scheme 8 Proposed reaction mechanism: (a) organo-redox-catalysis; (b) effective ET by direct outer-sphere ET or stepwise via $\mathrm{S}_{\mathrm{N}} 2$ reaction.

decomposed after $10 \mathrm{~h}$, in line with the reported 10 hours halflife temperature of $73{ }^{\circ} \mathrm{C}$ (red bottom line). ${ }^{41}$ Benzene and benzoic acid were formed in roughly equal amounts, indicating that half of the benzoyloxyl radicals had decarboxylated before they were quenched by HAT. Adding catalytic amounts (10 mol\%) of $\mathbf{A 1}$ accelerated the decomposition of BPO during the first 2 hours and resulted in forming predominantly benzoic acid (blue upper line). These results indicate that the amine catalyses the decomposition of BPO by an effective ET reaction (see Scheme $7 \mathrm{~b}$ below and its discussion for details), forming one molecule each of benzoate - observed as benzoic acid by NMR - and benzoyloxyl radical. The latter can decarboxylate to a phenyl radical and both radicals can react by HAT reactions from the medium to form benzoic acid and benzene, respectively. This explains the appearance of significantly more benzoic acid than benzene, compared to the thermal decomposition of BPO.

During the difunctionalization reaction, a white precipitate formed, which we found to be sodium benzoate, in line with the suggested cleavage of BPO by ET (see the ESI $\dagger$ ). All these results enable us to propose a mechanism for the formation of difunctionalization products like 6a (Scheme 8a) The peroxide bond of BPO is cleaved by an ET reaction from the triarylamine catalyst $\mathbf{A}$, forming the radical cation $\mathbf{A}+$, a benzoate anion and a benzoyloxyl radical. The presence of $\mathrm{NaPF}_{6}$ likely helps stabilizing the radical cation $\mathbf{A}+$ in the form of the salt $[\mathbf{A 1}+] \mathrm{PF}_{6}{ }^{-}$. The benzoyloxyl radical can engage with cyclohexane or other substrates in a HAT reaction, forming a carbon radical 14, which then adds to styrene, forming the benzylic radical $\mathbf{1 5}$. Oxidation by the ammoniumyl radical cation $\mathbf{A}+$ regenerates the triarylamine $\mathbf{A}$ and forms the carbocation 16, which is attacked by nucleophiles to provide the final product. To some extent, the benzoyloxyl radicals decarboxylate, generating phenyl radicals which can either participate in HAT reactions, too, or add to styrene, as was shown in Scheme $5 \mathrm{~b}$ above.

Whether the reaction between the triarylamine A and BPO proceeds by an outer-sphere ET, directly forming benzoate and two radicals, or by an $\mathrm{S}_{\mathrm{N}} 2$-reaction via $\mathrm{N}$-benzoyloxylammonium salt 17, which in a second step decomposes homolytically into the same products (Scheme $8 \mathrm{~b}$ ), is at present unclear. Both pathways have been suggested for reactions between diacylperoxides and amines, ${ }^{32,42-44}$ but the combination of BPO with the triarylamines used in this study has not been investigated yet.

Additionally, we studied the redox potentials of several triarylamines and the radical cation A1+ as well as BPO by cylic voltammetry (see the ESI $\dagger$ ). The reduction potential of $\mathbf{A 1}$ was indeed lower than that of BPO, supporting that $\mathbf{A 1}$ can reduce BPO by ET. However, BPO has the higher oxidation potential of the two, suggesting that it is the better electron acceptor. However, we consider these results of separate measurements as not fully conclusive for the interpretation of the mechanism, since they are not in agreement with the aforementioned results supporting catalysis by the amine. Furthermore, they do not take potential interactions in the reaction mixture into account. For example, the addition of $\mathrm{NaPF}_{6}$ is crucial for the reaction to occur, which indicates an ionic interaction that might shift the redox potential of the ammoniumyl radical cation. ${ }^{34}$ A species like 17 could be involved as electron acceptor, or the radical cation A1+ could be transformed into the actual catalyst in situ by attack at its free para-positions. ${ }^{21}$ Thus, we acknowledge that not all details of the present reactions are understood and we are therefore planning more detailed investigations.

\section{Conclusions}

In summary, we have established triarylamines as organoredox catalysts for oxidative $\mathrm{C}-\mathrm{H}$ functionalization reactions, with $p$-iodophenyl diphenylamine as the catalyst of choice in the newly developed method. By using benzoyl peroxide as oxidant, the difunctionalization of styrenes could be accomplished with radicals generated from hydrocarbons by hydrogen atom transfer and with nucleophiles, including nitriles, alcohols, acetic acid and fluoride. Besides, the amination of allylic and benzylic $\mathrm{C}-\mathrm{H}$ bonds is also achieved under the same reaction conditions. The method does not require irradiation, electrolysis, transition metals nor significantly elevated temperatures. This application of a relatively simple 
amine might pave the way for further developments of organoredox-catalysts, which may thus become another established class amongst organocatalysts. ${ }^{45}$

\section{Author contributions}

S.L. and M.K. conceived the project, M.K. supervised the project, S.L. executed all experiments, S.L. and M.K. composed the manuscript, S.L. composed the ESI.

\section{Conflicts of interest}

There are no conflicts to declare.

\section{Acknowledgements}

M. K. thanks the DFG (KL 2221/4-2, Heisenberg scholarship) and S. L. thanks the China Scholarship Council (CSC, doctoral scholarship no. 201808420290). We thank the analytical departments of the Max-Planck-Institut für Kohlenforschung for their support, Dr Jie Ouyang and Dr Qiang Cheng (MPI für Kohlenforschung) for help with cyclic voltammetry and Tobias Greven (Universität zu Köln) for supporting the reaction development. Open Access funding provided by the Max Planck Society.

\section{Notes and references}

$1 \mathrm{H}$. Yao, W. Hu and W. Zhang, Difunctionalization of Alkenes and Alkynes via Intermolecular Radical and Nucleophilic Additions, Molecules, 2021, 26, 105.

2 J.-S. Zhang, L. Liu, T. Chen and L.-B. Han, TransitionMetal-Catalyzed Three-Component Difunctionalizations of Alkenes, Chem. - Asian J., 2018, 13, 2277-2291.

3 X.-W. Lan, N.-X. Wang and Y. Xing, Recent Advances in Radical Difunctionalization of Simple Alkenes, Eur. J. Org. Chem., 2017, 5821-5851.

$4 \mathrm{H}$. Fischer and L. Radom, Factors Controlling the Addition of Carbon-Centered Radicals to Alkenes-An Experimental and Theoretical Perspective, Angew. Chem., Int. Ed., 2001, 40, 1340-1371.

5 M. Salamone and M. Bietti, Tuning Reactivity and Selectivity in Hydrogen Atom Transfer from Aliphatic $\mathrm{C}-\mathrm{H}$ Bonds to Alkoxyl Radicals: Role of Structural and Medium Effects, Acc. Chem. Res., 2015, 48, 2895-2903.

6 A. Bunescu, Q. Wang and J. Zhu, Copper-Catalyzed Cyanomethylation of Allylic Alcohols with Concomitant 1,2-Aryl Migration: Efficient Synthesis of Functionalized Ketones Containing an $\alpha$-Quaternary Center, Angew. Chem., Int. Ed., 2015, 54, 3132-3135.

7 N. Zhu, T. Wang, L. Ge, Y. Li, X. Zhang and H. Bao, $\gamma$-Amino Butyric Acid (GABA) Synthesis Enabled by Copper-
Catalyzed Carboamination of Alkenes, Org. Lett., 2017, 19, 4718-4721.

8 X.-H. Ouyang, Y. Li, R.-J. Song, M. Hu, S. Luo and J.-H. Li, Intermolecular dialkylation of alkenes with two distinct $\mathrm{C}-\mathrm{H}$ bonds enabled by synergistic photoredox catalysis and iron catalysis, Sci. Adv., 2019, 5, eaav9839.

9 M. Lux and M. Klussmann, Additions of Aldehyde-Derived Radicals and Nucleophilic N-Alkylindoles to Styrenes by Photoredox Catalysis, Org. Lett., 2020, 22, 3697-3701.

10 T. Koike and M. Akita, New Horizons of Photocatalytic Fluoromethylative Difunctionalization of Alkenes, Chem, 2018, 4, 409-437.

11 M.-Y. Cao, X. Ren and Z. Lu, Olefin difunctionalizations via visible light photocatalysis, Tetrahedron Lett., 2015, 56, 3732-3742.

12 X. Wang and A. Studer, Iodine(III) Reagents in Radical Chemistry, Acc. Chem. Res., 2017, 50, 1712-1724.

13 Y. Zheng, Y. He, G. Rong, X. Zhang, Y. Weng, K. Dong, $\mathrm{X}$. Xu and J. Mao, NaI-Mediated Acetamidosulphenylation of Alkenes with Nitriles as the Nucleophiles: A Direct Access to Acetamidosulfides, Org. Lett., 2015, 17, 54445447.

14 Q. Xue, J. Xie, P. Xu, K. Hu, Y. Cheng and C. Zhu, MetalFree, n-Bu4NI-Catalyzed Regioselective Difunctionalization of Unactivated Alkenes, ACS Catal., 2013, 3, 13651368.

15 J. C. Siu, N. Fu and S. Lin, Catalyzing Electrosynthesis: A Homogeneous Electrocatalytic Approach to Reaction Discovery, Acc. Chem. Res., 2020, 53, 547-560.

16 Y. Yuan, Y. Cao, Y. Lin, Y. Li, Z. Huang and A. Lei, Electrochemical Oxidative Alkoxysulfonylation of Alkenes Using Sulfonyl Hydrazines and Alcohols with Hydrogen Evolution, ACS Catal., 2018, 8, 10871-10875.

17 X. Jia, Radical Cation Salts: From Single-Electron Oxidation to C-H Activation, Synthesis, 2016, 48, 18-30.

18 J. Wang, K. Liu, L. Ma and X. Zhan, Triarylamine: Versatile Platform for Organic, Dye-Sensitized, and Perovskite Solar Cells, Chem. Rev., 2016, 116, 14675-14725.

19 R. Lartia, C. Allain, G. Bordeau, F. Schmidt, C. FioriniDebuisschert, F. Charra and M.-P. Teulade-Fichou, Synthetic Strategies to Derivatizable Triphenylamines Displaying High Two-Photon Absorption, J. Org. Chem., 2008, 73, 1732-1744.

20 Y. Shirota and H. Kageyama, Charge Carrier Transporting Molecular Materials and Their Applications in Devices, Chem. Rev., 2007, 107, 953-1010.

21 S. Dapperheld, E. Steckhan, K.-H. G. Brinkhaus and T. Esch, Organic Electron Transfer Systems, II Substituted Triarylamine Cation-Radical Redox Systems - Synthesis, Electrochemical and Spectroscopic Properties, Hammet Behavior, and Suitability as Redox Catalysts, Chem. Ber., 1991, 124, 2557-2567.

22 M. Thelakkat, Star-Shaped, Dendrimeric and Polymeric Triarylamines as Photoconductors and Hole Transport Materials for Electro-Optical Applications, Marcomol. Mater. Eng., 2002, 287, 442-461. 
23 W. Schmidt and E. Steckhan, Mild Oxidative Removal of the p-Methoxybenzyl Ether Protecting Group by Homogeneous Electron Transfer, Angew. Chem., Int. Ed. Engl., 1978, 17, 673-674.

24 N. L. Bauld, Cation radical cycloadditions and related sigmatropic reactions, Tetrahedron, 1989, 45, 5307-5363.

25 C.-Y. Cai and H.-C. Xu, Dehydrogenative reagent-free annulation of alkenes with diols for the synthesis of saturated O-heterocycles, Nat. Commun., 2018, 9, 3551.

26 X. Wu, A. P. Davis and A. J. Fry, Electrocatalytic Oxidative Cleavage of Electron-Deficient Substituted Stilbenes in Acetonitrile-Water Employing a New High Oxidation Potential Electrocatalyst. An Electrochemical Equivalent of Ozonolysis, Org. Lett., 2007, 9, 5633-5636.

27 L. Wang, R. Li and K. A. I. Zhang, Atom Transfer Radical Polymerization (ATRP) Catalyzed by Visible Light-Absorbed Small Molecule Organic Semiconductors, Macromol. Rapid Commun., 2018, 39, 1800466.

28 L. Wang, J. Byun, R. Li, W. Huang and K. A. I. Zhang, Molecular Design of Donor-Acceptor-Type Organic Photocatalysts for Metal-free Aromatic C-C Bond Formations under Visible Light, Adv. Synth. Catal., 2018, 360, 4312-4318.

29 X. Jia, F. Peng, C. Qing, C. Huo and X. Wang, Catalytic Radical Cation Salt Induced Csp3-H Functionalization of Glycine Derivatives: Synthesis of Substituted Quinolines, Org. Lett., 2012, 14, 4030-4033.

30 F. Unglaube, P. Hünemörder, X. Guo, Z. Chen, D. Wang and E. Mejía, Phenazine Radical Cations as Efficient Homogeneous and Heterogeneous Catalysts for the CrossDehydrogenative Aza-Henry Reaction, Helv. Chim. Acta, 2020, 103, e2000184.

31 A. Székely and M. Klussmann, Molecular radical chain initiators for ambient to low temperature applications, Chem. - Asian J., 2019, 14, 105-115.

32 K. Kim, N. R. Singstock, K. K. Childress, J. Sinha, A. M. Salazar, S. N. Whitfield, A. M. Holder, J. W. Stansbury and C. B. Musgrave, Rational Design of Efficient Amine Reductant Initiators for Amine-Peroxide Redox Polymerization, J. Am. Chem. Soc., 2019, 141, 62796291.

33 E. Boess, M. V. Hoof, S. L. Birdsall and M. Klussmann, Investigating the Oxidation Step in the CuCl2-Catalyzed Aerobic Oxidative Coupling Reaction of N-Aryl
Tetrahydroisoquinolines, J. Org. Chem., 2020, 85, 19721980.

34 S. Liu and M. Klussmann, Acid Promoted Radical-Chain Difunctionalization of Styrenes with Stabilized Radicals and (N,O)-Nucleophiles, Chem. Commun., 2020, 56, 15571560.

35 R. K. Neff, Y.-L. Su, S. Liu, M. Rosado, X. Zhang and M. P. Doyle, Generation of Halomethyl Radicals by Halogen Atom Abstraction and Their Addition Reactions with Alkenes, J. Am. Chem. Soc., 2019, 141, 16643-16650.

36 T. Constantin, M. Zanini, A. Regni, N. S. Sheikh, F. Juliá and D. Leonori, Aminoalkyl radicals as halogen-atom transfer agents for activation of alkyl and aryl halides, Science, 2020, 367, 1021-1026.

37 C. Chatalova-Sazepin, Q. Wang, G. M. Sammis and J. Zhu, Copper-Catalyzed Intermolecular Carboetherification of Unactivated Alkenes by Alkyl Nitriles and Alcohols, Angew. Chem., Int. Ed., 2015, 54, 5443-5446.

38 T. Jun, W. Hiroyasu, M. Masako and K. Nobuaki, Titanocene-Catalyzed Alkylation of Aryl-Substituted Alkenes with Alkyl Halides, Bull. Chem. Soc. Jpn., 2003, 76, 22092214.

39 B. J. Fallon, V. Corcé, M. Amatore, C. Aubert, F. Chemla, F. Ferreira, A. Perez-Luna and M. Petit, A well-defined lowvalent cobalt catalyst $\mathrm{Co}(\mathrm{PMe} 3) 4$ with dimethylzinc: a simple catalytic approach for the reductive dimerization of benzyl halides, New J. Chem., 2016, 40, 9912-9916.

40 A. Studer and D. P. Curran, Catalysis of Radical Reactions: A Radical Chemistry Perspective, Angew. Chem., Int. Ed., 2016, 55, 58-102.

41 C. S. Sheppard, in Encyclopedia of polymer science and engineering, John Wiley \& Sons, Inc., 1985, vol. 11, pp. 1-21.

42 N. A. Turovskii, I. A. Opeida and O. V. Kushch, Intermediates in Reactions of Diacyl Peroxides with Tertiary Aliphatic Amines, Russ. J. Org. Chem., 2003, 39, 642-645.

43 S. Srinivas and K. G. Taylor, Amine-induced reactions of diacyl peroxides, J. Org. Chem., 1990, 55, 1779-1786.

44 D. B. Denney and D. Z. Denney, Studies of the Mechanisms of the Reactions of Benzoyl Peroxide with Secondary Amines and Phenols, J. Am. Chem. Soc., 1960, 82, 13891393.

45 D. W. C. MacMillan, The advent and development of organocatalysis, Nature, 2008, 455, 304-308. 